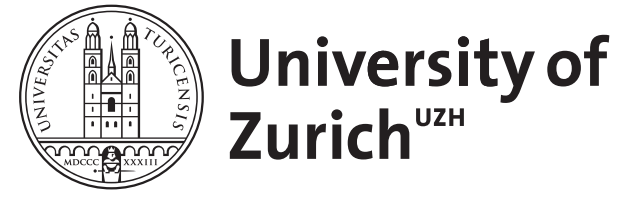
Archive

University of Zurich

University Library

Strickhofstrasse 39

CH-8057 Zurich

www.zora.uzh.ch

Year: 2002

\title{
Kommentar zu L. Wilhelm Weber
}

Frey, Bruno S

DOI: https://doi.org/10.1111/1468-2516.00079

Posted at the Zurich Open Repository and Archive, University of Zurich

ZORA URL: https://doi.org/10.5167/uzh-155090

Journal Article

Published Version

Originally published at:

Frey, Bruno S (2002). Kommentar zu L. Wilhelm Weber. Perspektiven der Wirtschaftspolitik:n/a. DOI: https://doi.org/10.1111/1468-2516.00079 


\title{
Kommentar zu L. Wilhelm Weber
}

\author{
Bruno S. Frey \\ Universität Zürich
}

Für den ausführlichen Kommentar von L. Wilhelm Weber zu meinem Beitrag „Was bewirkt die Volkswirtschaftslehre“ in der ersten Nummer der Wirtschaftspolitischen Perspektiven bin ich dankbar. Er stellt eine wichtige Ergänzung anhand praktischer wirtschaftspolitischer Ereignisse in Deutschland dar. Derartige Schilderungen der Wirtschaftspolitik erlauben der Wirtschaftswissenschaft besser zu erkennen, in welcher Weise theoretische Erkenntnisse aufgenommen oder vernachlässigt werden. Vor allem wird der Blick von der üblicherweise betrachteten Entscheidung zwischen vorgegebenen alternativen wirtschaftspolitischen Massnahmen auf den Prozess der Entscheidungsbildung gelenkt, zum Beispiel also, welche Argumente in welcher Phase der Entscheidung als zulässig erachtet werden und welches Gewicht sie haben.

Auch wenn L. Wilhelm Webers Bericht wichtige Einsichten liefern kann, trifft er doch nicht den Kern meines Beitrags. Ich bezweifle nicht, dass ein Teil der Volkswirtschaftslehre wirtschaftspolitische Ratschläge anbieten kann und dass Teile der Ökonomik von Ministerialbeamten und Politikern aufgenommen werden. Allerdings handelt es sich um einen zunehmend kleineren Teil. Meiner Beobachtung nach wird die Volkswirtschaftslehre immer weniger im Sinne einer Auseinandersetzung mit den Problemen der Gegenwart verstanden. Vielmehr geht es zunehmend um die Lösung abgekoppelter, wissenschaftsinterner logischer Puzzles, die kaum - und häufig gar nicht - auf Anforderungen zur Bewältigung gesellschaftlicher Probleme zurückgehen. Dies gilt auch für manche empirische Untersuchungen mittels ökonometrischer Methoden, bei den zwar Zusammenhänge ermittelt werden, es aber völlig offen bleibt, wozu sie dienen. Die Ökonominnen und Ökonomen werden deshalb immer weniger fähig und willens, sich den Herausforderungen der Wirtschaftspolitik zu stellen. Wer dies bezweifelt, sollte sich die Ausrichtung des wissenschaftlichen Nachwuchses gerade der als führend geltenden Universitäten ansehen.

Herr Weber unterscheidet in seinen Ausführungen meines Erachtens zu wenig zwischen wirtschaftspolitischer Beratung und wirtschaftspolitischen Entscheidungen einerseits - beides sind nur Inputs - und den tatsächlichen Auswirkungen auf die wirtschaftliche Tätigkeit - dem Output - andererseits. Offensichtlich wird wirtschaftspolitischer Rat oft nicht aufgenommen,

(C) Verein für Socialpolitik und Blackwell Publishers Ltd. 2002, 108 Cowley Road, Oxford OX4 1JF, UK und 350 Main Street, Malden, MA 02148, USA. 


\section{Bruno S. Frey}

zumindest nicht derart, dass vom ökonomischen Gehalt viel übrig bleibt. Aber selbst wenn aus einem Rat wirtschaftspolitische Entscheidungen folgen, ist nicht gezeigt, dass die wirtschaftliche Tätigkeit tatsächlich beeinflusst wurde. Es genügt auf drei Aspekte hinzuweisen:

(1) Die Politiker hätten ohnehin getan, was von der Ökonomik vorgeschlagen wird;

(2) Die wirtschaftspolitische Entscheidung vollzieht nur nach, was ohnehin in der Wirtschaft geschehen wäre;

(3) Private Akteure oder andere politische Entscheidungsträger heben die wirtschaftlichen Effekte durch entsprechendes Verhalten auf (worauf das Konzept der „rationalen Erwartungen“ hinweist).

Diese Aspekte treffen natürlich nicht immer zu. Mein Beitrag sollte als Anregung verstanden werden, sich mit der Ökonomik der Ökonomik zu befassen. Es sollte nicht einfach apodiktisch von einem (starken) Einfluss der Volkswirtschaftslehre auf die wirtschaftliche Aktivität ausgegangen werden. Vielmehr ist empirisch (ökonometrisch) zu untersuchen, welche Elemente der Ökonomik präzis das wirtschaftliche Geschehen beeinflusst haben. 